Portland State University

PDXScholar

Systems Science Faculty Publications and

Presentations

Systems Science

$7-29-2020$

\title{
Joint Lattice of Reconstructability Analysis and Bayesian Network General Graphs
}

\author{
Marcus Harris \\ Portland State University \\ Martin Zwick \\ Portland State University, zwick@pdx.edu
}

Follow this and additional works at: https://pdxscholar.library.pdx.edu/sysc_fac

Part of the Logic and Foundations Commons, and the Systems Architecture Commons Let us know how access to this document benefits you.

\section{Citation Details}

Harris, Marcus and Zwick, Martin (2020). "Joint Lattice of Reconstructability Analysis and Bayesian Network General Graphs." International Conference on Complex Systems (New England Complex Systems Institute), On-line. July 29.

This Presentation is brought to you for free and open access. It has been accepted for inclusion in Systems Science Faculty Publications and Presentations by an authorized administrator of PDXScholar. Please contact us if we can make this document more accessible: pdxscholar@pdx.edu. 


\title{
Joint Lattice of Reconstructability Analysis and Bayesian Network General Graphs
}

\author{
Marcus Harris ${ }^{(凶)}$ and Martin Zwick \\ Portland State University, Portland, Oregon, USA \\ maharris@pdx.edu, zwick@pdx.edu
}

\begin{abstract}
This paper integrates the structures considered in Reconstructability Analysis (RA) and those considered in Bayesian Networks (BN) into a joint lattice of probabilistic graphical models. This integration and associated lattice visualizations are done in this paper for four variables, but the approach can easily be expanded to more variables. The work builds on the RA work of Klir (1985), Krippendorff (1986), and Zwick (2001), and the BN work of Pearl (1985, 1987, 1988, 2000), Verma (1990), Heckerman (1994), Chickering (1995), Andersson (1997), and others. The RA four variable lattice and the BN four variable lattice partially overlap: there are ten unique RA general graphs, ten unique BN general graphs, and ten general graphs common to both RA and BN. For example, the specific graph having probability distribution $\mathrm{p}(\mathrm{A}) \mathrm{p}(\mathrm{C}) \mathrm{p}(\mathrm{B} \mid \mathrm{AC})$ is unique to $\mathrm{BN}$, the RA specific graph $\mathrm{AB}: \mathrm{AC}: \mathrm{BC}$, which contains a loop, is unique to RA, and the specific graph ACD:BCD with probability distribution $\mathrm{p}(\mathrm{A} \mid \mathrm{CD}) \mathrm{p}(\mathrm{B} \mid \mathrm{CD}) \mathrm{p}(\mathrm{D} \mid \mathrm{C}) \mathrm{p}(\mathrm{C})$ is common to both $\mathrm{RA}$ and $\mathrm{BN}$. The joint RA-BN lattice of general graphs presented in this paper expands the set of general graphs with unique independence structures beyond what was previously available by either RA alone or BN alone, thus allowing for representations of complex systems which are (i) more accurate relative to data and/or (ii) simpler and thus more comprehensible and more generalizable than would be possible by modeling only with RA or only with BN.
\end{abstract}

\section{Introduction}

Reconstructability Analysis (RA) is a data modeling approach developed in the systems community (Ashby, 1964; Klir, 1976, 1985, 1986; Conant, 1981, 1988; Krippendorff, 1981, 1986; Broekstra, 1979; Cavallo, 1979; Zwick, 2001, 2004; and others) that combines graph theory and information theory. Its applications are diverse, including time-series analysis, classification, decomposition, compression, pattern recognition, prediction, control, and decision analysis (Zwick, 2004). It is designed especially for nominal variables, but continuous variables can be accommodated if their values are discretized. Graph theory specifies the structure of the model (the set of relations between the variables), which is independent of actual data (except for specification of variable cardinalities); information theory uses the data to characterize the strength and the precise nature of the relations. Data applied to a graph structure yields a probabilistic graphical model of the data.

In RA, graphs are undirected, although directions are implicit if one variable is designated as the response variable (dependent variable), while all other variables are

Harris, Marcus and Zwick, Martin (2020). International Conference on Complex Systems (New England Complex Systems Institute), On-line. July 29. 
designated as explanatory variables (independent variables). In this paper, such IVDV designations are not made, so we are concerned with only what are called "neutral systems." Graphs are either general or specific. A general graph identifies relations among variables that are unlabeled; a specific graph labels the variables. For example, for a system consisting of variables $\mathrm{A}, \mathrm{B}$, and $\mathrm{C}, \mathrm{AB}: \mathrm{BC}$ is a specific graph where nodes $\mathrm{A}$ and $\mathrm{B}$ are linked and $\mathrm{B}$ and $\mathrm{C}$ are also linked. In this notation, the order of variables in any relation is arbitrary, as is the order of the relations. Relations include all of their embedded relations. For example, the relation $\mathrm{ABC}$ includes embedded relations $\mathrm{AB}, \mathrm{AC}$ and $\mathrm{BC}$ and the univariate margins $\mathrm{A}, \mathrm{B}$, and $\mathrm{C}$. Specific graphs $\mathrm{AB}: \mathrm{BC}, \mathrm{BA}: \mathrm{AC}$ and $\mathrm{AC}: \mathrm{CB}$ are all instances of the same general graph that contains a unique independence structure regardless of variable labels.

For a three variable neutral system with loops there are 5 general graphs and 9 specific graphs; for four variables there are 20 general graphs and 114 specific graphs. The number of graphs increases hyper-exponentially with the number of variables.

A Bayesian Network (BN) is another graphical modeling approach for data modeling that is closely related to RA; indeed where it overlaps RA the two methods are equivalent, but RA and BN each has distinctive features absent in the other methodology. BNs have origins in the type of path model originally described by Wright (1921, 1934), but it was not until the 1980s that BNs were more formally established (Pearl, 1985, 1987, 1988; Neapolitan, 1989).

As does RA, BN combines graph theory and probability theory; graph theory provides the structure and probability theory characterizes the nature of relationships between variables. BNs are represented by a single type of graph structure; a directed acyclic graph, which is a subset of chain graphs, also known as block recursive models (Lauritzen, 1996). For a three variable BN lattice, there are 5 general graphs and 11 specific graphs; for four variables there are 20 general graphs and 185 specific graphs.

This paper integrates RA and $\mathrm{BN}$ and visualizes their joint lattice of general graphs for four variables.

\section{RA Lattice}

The lattice of four variable RA general graphs of Figure 1 (adapted from Klir, 1985; Krippendorff, 1986) represents all four variable RA graphs with unique independence structures. In these graphs, lines (which can branch) are variables; boxes are relations between variables. Where only two lines extend from a box, the relation is dyadic. If more than two lines extend from a box, the graph is a hypergraph. Bolded general graphs in Figure 1 are acyclic whereas non-bolded general graphs have cycles. The figure shows all 20 general graphs for four variables. G1 is the most complex general graph, in which all four variables are connected in a tetradic relation. Graphs below G1 are increasingly less complex decompositions of G1, ending with G20, the least complex graph, representing complete independence among all four variables. 


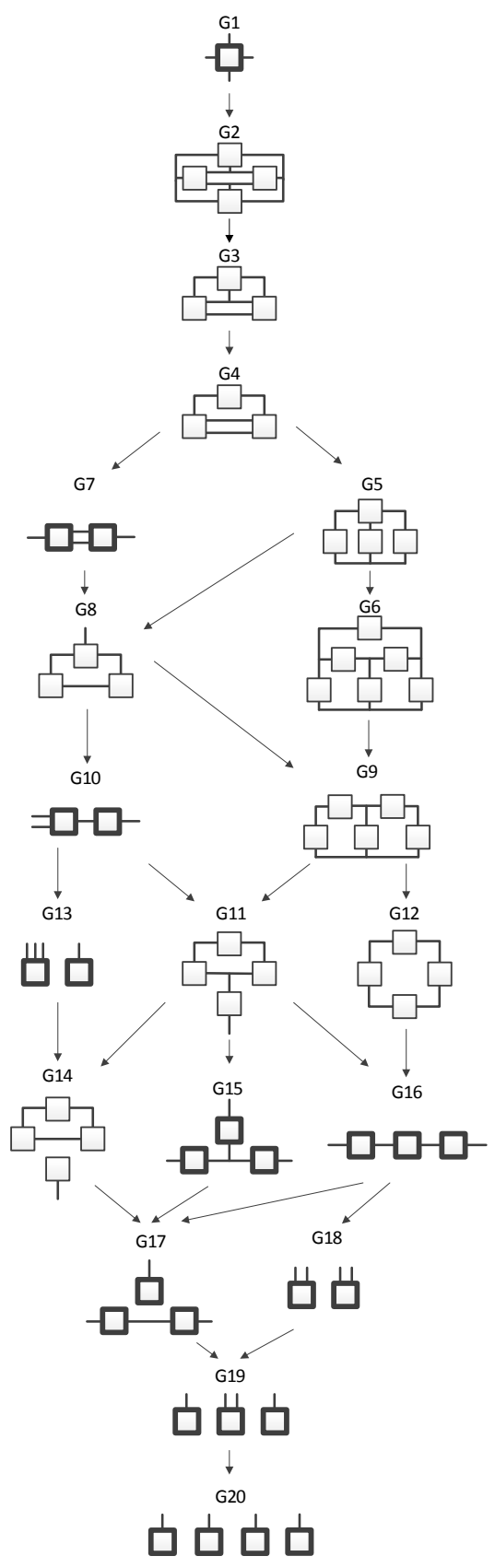

Fig. 1. RA General Graph Lattice 
A general graph represents a unique independence structure which disregards all possible ways that variables could be labeled. For example, the left-most and right-most variables in G7 in Figure 1 are independent of one another given the two central variables that connect both relations, where these four variables have not been assigned any specific identities.

When, in RA or BN, the variables of a general graph are labeled, it is called a specific graph equivalence class or specific graph and is synonymous with a Markov equivalence class (Andersson, 1997). A specific graph, given data, produces a unique probability distribution. (This is the case for RA; as explained below, two different directed specific BN graphs can have the same distribution.) Since the number of graphs increases hyper-exponentially with the number of variables, searching the entire lattice for the best model can be very computationally expensive as the number of variables increases.

The colon notation for RA specific graphs represents marginal or conditional independence between variables. For example, G20 from Figure 1, having notation

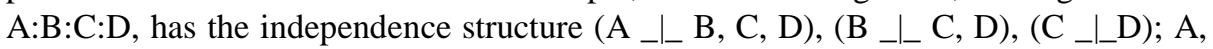
$\mathrm{B}, \mathrm{C}$, and $\mathrm{D}$ are all marginally independent of each other. G17, having notation $\mathrm{AB}: \mathrm{BC}: \mathrm{D}$, has the independence structure ( $\left.\mathrm{D}_{-} \mathrm{L}_{-} \mathrm{A}, \mathrm{B}, \mathrm{C}\right),\left(\mathrm{A} \_-\mathrm{C} \mid \mathrm{B}\right)$; $\mathrm{A}, \mathrm{B}$, and $\mathrm{C}$ are all marginally independent of $\mathrm{D}$, and $\mathrm{A}$ is conditionally independent of $\mathrm{C}$ given $\mathrm{B}$. The overlap of $\mathrm{B}$ in the $\mathrm{AB}$ and $\mathrm{BC}$ relations encodes the conditional independence of $\mathrm{A}$ and $\mathrm{C}$ given $\mathrm{B}$.

\section{BN Lattice}

The primary differences between RA and BN are two-fold: (1) BNs are directed and acyclic whereas RA graphs are undirected and can be cyclic or acyclic, and (2) some $\mathrm{BN}$ graphs contain converging edges, called a V-structure, which encodes a unique independence structure not found in RA general graphs.

As in RA, there are BN general graphs and BN specific graphs, in the BN literature referred to as essential graphs and Markov equivalence classes respectively (Anderson, 1997). BN general graphs of Figure 2 represent unique independence structures of variables, edges, and edge orientations, where specific identities are not assigned to the variables. Figure 2 shows the hierarchy of BN general graphs for four variables. There are $20 \mathrm{BN}$ general graphs in the lattice, i.e., 20 unique general independence structures for four variable BNs.

In Figure 2, general graphs are labeled BN1, BN2...BN19, BN20. Solid squares represent variables, edges are represented by directed arrows from one square to another. The dashed lines with arrows from one general graph to another represent the hierarchy of general graphs, with parent graphs being above child graphs. Child graphs result from the deletion of one edge from the parent graph. The bottom-right addition to the lattice that tabulates equivalent general graphs is discussed in 3.2. 


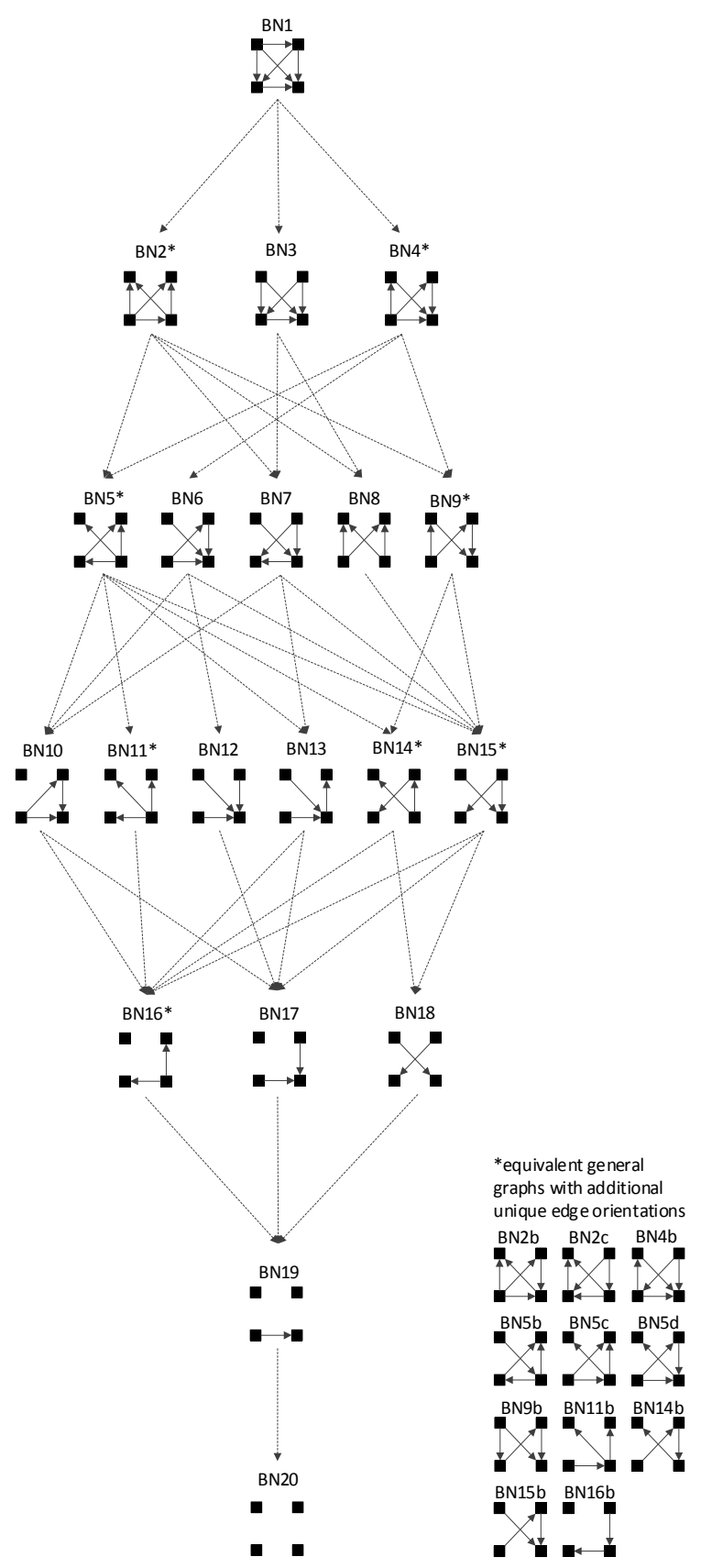

Fig. 2. BN General Graph Lattice 
The alphabetic notation that we use for BN specific graphs is derived from the RA notation described previously. For a BN graph without a V-structure, the notation is identical to the RA notation. As in RA, the colon represents marginal or conditional independence among variables. For a BN graph with a V-structure, the notation adds subscripts to represent the independence relations encoded by the V-structure, which are unique to BNs and not found in RA. For example, BN17 from Figure 2, with labels A, B, C, D in order of top left, top right, bottom left, bottom right respectively, is given the notation $\mathrm{BCD}_{\mathrm{B}: \mathrm{C}}: \mathrm{A}$, where the colon between $\mathrm{BCD}_{\mathrm{B}: \mathrm{C}}$ and $\mathrm{A}$ states the independency ( $\left.A_{-}\right|_{-} B, C, D$ ), namely that $A$ is marginally independent of $B, C$, and $D$. The subscript ${ }_{\mathrm{B}: \mathrm{C}}$ encodes marginal independence between $\mathrm{B}$ and $\mathrm{C}$ within the triadic $\mathrm{BCD}$ relation.

\subsection{Generating the lattice of BN general and specific graphs}

The BN literature on lattices predominately focuses on search algorithms to find the best $\mathrm{BN}$ given a scoring metric. Implicit in these search algorithms is a lattice of candidate graphs being explored in search of the best model. Chickering (2004) and others have shown the search problem to be NP-hard, with four variables there are 543 possible BNs, with 10 variables there are $\mathrm{O}\left(10^{\wedge} 18\right)$ (Murphy, 1998). Because of this, the BN literature has focused less on the description of the exhaustive lattice of BN structures, and more on advancing search heuristics to efficiently identify the best BN given a scoring metric (Buntine, 1991a, 1991b; Cooper, 1992; Bouckaert, 1994; Heckerman, 1994; Chickering, 1995, 1997; Friedman, 1996, 2003; Larranaga, 1996; Koivisto, 2004; Malone 2011; and others).

Heckerman (1994) first showed that BNs with differing edge topologies can have the same independence structure and thus the same probability distribution, herein described as specific graphs. In contrast to heuristics that search all BNs, search heuristics for $\mathrm{BN}$ specific graphs have proven to be more efficient because they reduce the dimensionality of search space (Chickering, 1995, 2002; Andersson, 1997; Gillispie, 2001; Studený, 2010; Tian, 2010; Zhang, 2012; Chen 2016; and others). For four variables, this reduces the search space from 543 BNs to 185 specific graphs (Anderson, 1997). Removing variable labels, these specific graphs can be summarized by 20 general graphs.

Building from the RA work of Klir (1986) and Zwick (2001), and the BN work of Pearl (1985, 1987, 1988, 2000), Verma (1990), Heckerman (1994), Chickering (1995), Andersson (1997), and others, the following procedure was used to generate the four variable BN general and specific graph lattice in a way that can be integrated with the four variable RA general graph lattice. While this procedure is applied in this paper to four variables, it could be used for any number of variables. 
Procedure to generate the $\mathrm{BN}$ general and specific graph lattice for any number of variables

1. Generate all graphs for $n$ variables by permuting all possible variable labels, edge connections and edge orientations. Eliminate any graphs with loops. The result is the set of all directed acyclic graphs for $n$ variables.

2. For each directed acyclic graph, evaluate the specific independence structure following the d-separation procedure (MIT, 2015) to generate the exhaustive list of independencies for each graph.

3. Organize graphs with the same labeled independencies into specific graph equivalence classes.

4. From each specific graph equivalence class, select a single edge topology to represent the general graph equivalence class. List any additional equivalent general graphs with unique edge topologies separately, as done in Figure 2.

5. Organize general graphs into levels based upon the number of edges in each general graph and link nested general graphs in the lattice to reflect parentchild general graphs.

\subsection{Additional representations of $\mathrm{BN}$ general graph equivalence classes}

There are 20 general graphs in the four variable BN lattice. However eight of these, marked with asterisks in Figure 2, can be represented by additional unique edge topologies which, however, result in identical probability distributions when applied to data. These are BN2*, BN4*, BN5*, BN9*, BN11*, BN14*, BN15*, and BN16*. Thus, for example, BN2b has edge orientations that are different from (and cannot be mapped onto) those of BN2*, but when variables are labeled in BN2* and BN2b, identical probability distributions result. This property is unique to BN and is not found in RA, in which there is a single unique representation of each RA general graph.

\section{$4 \quad$ Integrating the Rho, RA and BN Lattices}

This section integrates the RA and BN general graph lattices using the four variable Rho lattice (Klir, 1985). The joint RA-BN lattice of general graphs presented in this paper expands the set of general graphs with unique independence structures beyond what was previously available by either RA alone or BN alone. The lattice identifies general independence structures unique to RA, general independence structures unique to BNs, and general independence structures that are equivalent across RA and BNs. Where two or more RA or BN graphs have the same general independence structure regardless of variable labels, they are equivalent. 


\subsection{Lattice of four variable Rho graphs}

The four variable Rho $(\rho)$ lattice of Figure 3 (adapted from Klir, 1985, p. 237) is a simplification of the RA lattice of general graphs of Figure 1. The Rho lattice represents all possible undirected relations between four variables, an even more general lattice than that of the RA general graph lattice and general enough to map both RA and BN four variable general graphs to one of the eleven represented structures. Solid dots represent variables; lines connecting dots represent relations between variables. In terms of the RA general graph lattice of Figure 1, if two variables are directly connected by any box (a relation of arbitrary ordinality), a line connects them in the Rho lattice. Arrows from one Rho graph to another represent hierarchy, i.e., the generation of a child graph from a parent graph. The graph $\rho 1$ represents maximal connectedness, or interdependence, between variables, and the graph $\rho 11$ represents independence among all variables. Graphs in-between $\rho 1$ and $\rho 11$ represent a mix of dependence and independence among variables. Each RA or $\mathrm{BN}$ graph corresponds to one, and only one, of the eleven Rho graphs.

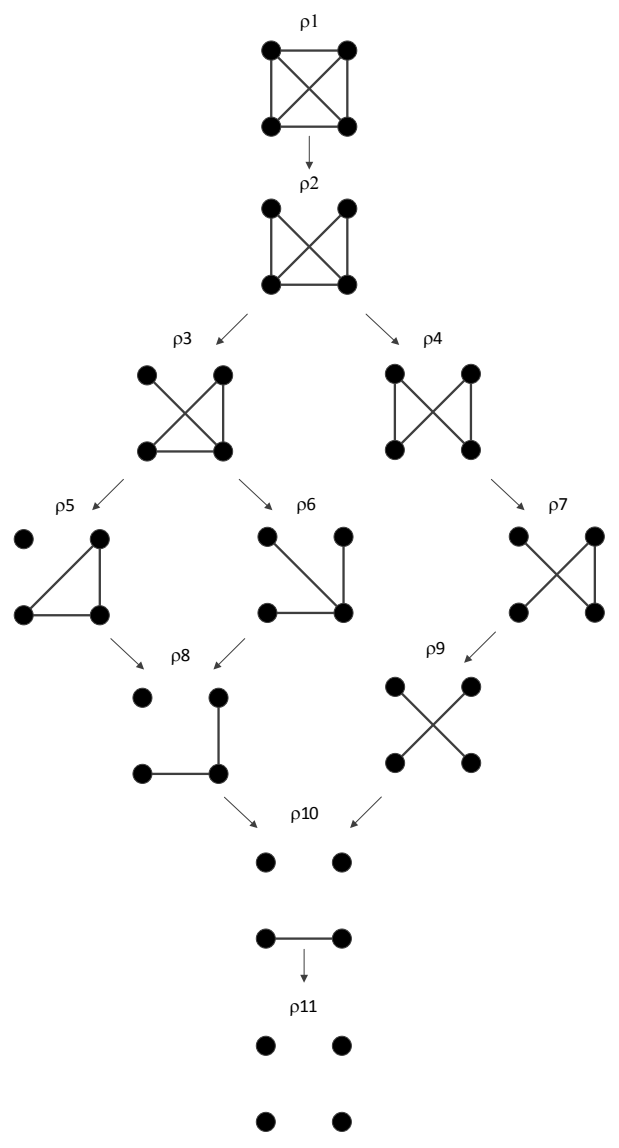

Fig. 3. Rho Lattice

\subsection{Equivalent RA and BN general graphs}

Out of 20 RA general graphs and 20 BN general graphs, there are 10 RA general graphs, comprising all of the acyclic graphs in the RA lattice that are equivalent to BN general graphs. Each of these RA-BN equivalent pairs corresponds to one of the 11 Rho graphs from Figure 3, with the exception of $\rho 4$. $\rho 4$ has corresponding RA and $\mathrm{BN}$ general graphs, but these do not have equivalent independence structures, and are discussed in the following section on non-equivalent RA and BN general graphs. 
Figure 4 shows an example of equivalent RA and BN graphs, namely G7 and BN2*, respectively. Labeled variables in $G 7$ results in independencies $\left(A_{-}{ }_{-} B \mid C\right.$, D) and thus the RA specific graph notation ACD:BCD. Assigning labels to BN2* yields the same independencies and thus the same specific graph ACD:BCD.
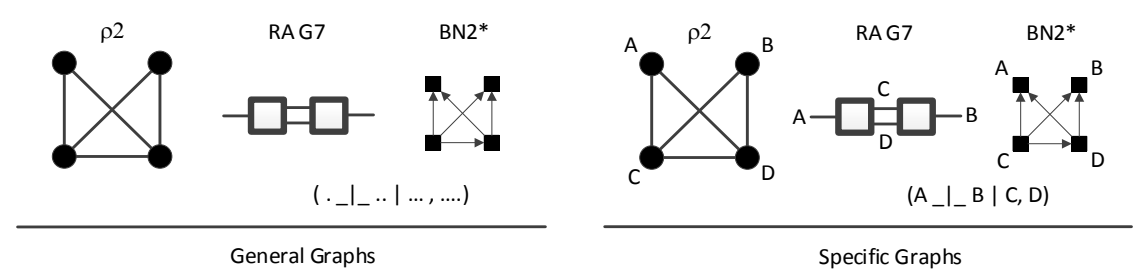

Fig. 4. RA G7 and BN2* general and specific graph example

Table 1 shows the list of all equivalent Rho, RA and BN four variable general graphs, an example of their specific graph notation, and their independences. These specific graph examples align with the $\mathrm{BN}$ general graphs of Figure 2 assuming labeling of nodes A, B, C, D in the order of top left, top right, bottom left, bottom right.

Table 1. Rho, RA and BN equivalent graphs

\begin{tabular}{|c|c|c|c|c|}
\hline Rho graph & $\begin{array}{l}\text { RA general } \\
\text { graph }\end{array}$ & $\begin{array}{l}\text { BN general } \\
\text { graph }\end{array}$ & $\begin{array}{l}\text { Specific Graph } \\
\text { Example } \\
\text { (RA notation) }\end{array}$ & Independencies \\
\hline$\rho 1$ & G1 & BN1 & $A B C D$ & no independencies \\
\hline$\rho 2$ & G7 & BN2* & $A C D: B C D$ & $\left(A_{-} I_{-} B \mid C, D\right)$ \\
\hline$\rho 3$ & G10 & BN5* & $B C D: A D$ & $\left(A \_\left|\_B, C\right| D\right)$ \\
\hline$\rho 5$ & G13 & $\mathrm{BN} 10$ & BCD:A & $\left(A_{-} I_{-} B, C, D\right)$ \\
\hline$\rho 6$ & G15 & BN11* & $A D: B D: C D$ & $\left(A \_\left|\_B, C\right| D\right),\left(B \_|C C| D\right)$ \\
\hline$\rho 7$ & G16 & BN14* & $A D: B C: B D$ & $\left(A \_\left|\_B\right| D\right),\left(C \_I \_A, D \mid B\right)$ \\
\hline$\rho 8$ & G17 & BN16* & BD:CD:A & $\left(B \_I \_C \mid D\right),\left(A \_\mid \_B, C, D\right)$ \\
\hline$\rho 9$ & G18 & BN18 & $A D: B C$ & $\left(A, D \_I_{-} B, C\right)$ \\
\hline$\rho 10$ & G19 & BN19 & $C D: A: B$ & $\left(B \_I \_C, D\right),\left(A \_I \_B, C, D\right)$ \\
\hline$\rho 11$ & G20 & BN20 & $A: B: C: D$ & $\left(A \_I \_B, C, D\right),\left(B \_I \_C, D\right),\left(C \_I \_D\right)$ \\
\hline
\end{tabular}

\subsection{Non-equivalent $\mathrm{RA}$ and $\mathrm{BN}$ general graphs}

In addition to the 10 equivalent $\mathrm{RA}$ and $\mathrm{BN}$ general graphs, there are 10 general graphs unique to the RA lattice and 10 general graphs unique to the BN lattice. All 10 non-equivalent RA general graphs in the four variable lattice are cyclic and require iteration to generate their probability distributions. BNs are acyclic and have analytic solutions, so there are no BN graphs that are equivalent to these cyclic RA graphs. Since cyclic RA graphs are undirected, one might think that there could be some equivalent directed acyclic BN graphs, but this is not the case, because BN graphs that are acyclic when directions are considered but cyclic if directions are ignored have V-structure interpretations, as described previously. All 10 non-equivalent BN graphs have such $\mathrm{V}$-structures, which encode independence relations unique to BNs. 
To illustrate: the structure $\mathrm{A} \rightarrow \mathrm{B}, \mathrm{B} \rightarrow \mathrm{C}, \mathrm{C} \rightarrow \mathrm{D}, \mathrm{D} \rightarrow \mathrm{A}$ is cyclic and not a legitimate $\mathrm{BN}$ structure, but the directed structure of $\mathrm{A} \rightarrow \mathrm{B}, \mathrm{B} \rightarrow \mathrm{C}, \mathrm{C} \rightarrow \mathrm{D}, \mathrm{A} \rightarrow \mathrm{D}$, which has the same undirected links, is not cyclic, and is a legitimate BN structure, namely BN9b. However, this latter structure is not interpreted as a set of dyadic relations, which would be written in RA notation as AB:BC:CD:AD. Rather, the V-structure consisting of $\mathrm{C} \rightarrow \mathrm{D}$ and $\mathrm{A} \rightarrow \mathrm{D}$ is interpreted as a triadic relation, which contributes a $\mathrm{p}(\mathrm{D} \mid \mathrm{AC})$ to the probability expression, $\mathrm{p}(\mathrm{A}) \mathrm{p}(\mathrm{B} \mid \mathrm{A}) \mathrm{p}(\mathrm{C} \mid \mathrm{B}) \mathrm{p}(\mathrm{D} \mid \mathrm{AC})$, which does not correspond to any RA structure.

\subsection{Joint lattice of RA and BN general graphs}

The joint lattice of RA and BN general graphs is organized using the Rho lattice (Klir, 1985) of Figure 3, the RA general lattice of Figure 1 (Klir 1985, Krippendorff 1986) and the BN general lattice of Figure 2. All 20 RA general graphs and all $20 \mathrm{BN}$ general graphs for each Rho graph are shown in the joint lattice of RA and BN general graphs of Figure 5.

In Figure 5, within each Rho graph, where RA and BN general graphs are equivalent, that is, their independence structures are identical, the $\mathrm{BN}$ graph is placed underneath the RA equivalent graph. Where RA or BN graphs are not equivalent, representing an independence structure unique to RA or to BN, they stand alone side-by-side. Arrows from one graph to another in the joint lattice represent the hierarchy of the RA lattice only.

\section{Conclusions}

The joint lattice of RA and BN general graphs for four variables increases the number of general graphs with unique independence structures from 20 in the four variable RA lattice and 20 in the four variable BN lattice to 30 in the joint RA-BN lattice, and when variable labels are added, increases the number of unique specific graphs from 114 in the RA lattice and 185 in the BN lattice to 238 in the joint lattice.

The integration of the two lattices offers a richer and more expansive way to model complex systems leveraging the $\mathrm{V}$-structure unique to $\mathrm{BN}$ graphs and the allowability of cycles in RA graphs. The joint RA-BN lattice of general graphs presented in this paper expands the set of general graphs with unique independence structures (or, equivalently, with unique interdependence structures) beyond what was previously available by either RA alone or BN alone, thus allowing for representations of complex systems which are (i) more accurate relative to data and/or (ii) simpler and thus more comprehensible and more generalizable than would be possible by modeling only with RA or only with BN. This joint lattice thus demonstrates how these two related frameworks - RA and BN - both members of the family of probabilistic graphical modeling methodologies, can be integrated into a unified framework. Extension of this work will include designing algorithms to search this joint RA-BN lattice, analysis of RA and BN predictive models in which the IV-DV distinction is made, consideration of "hybrid" RA-BN models, and other topics. 

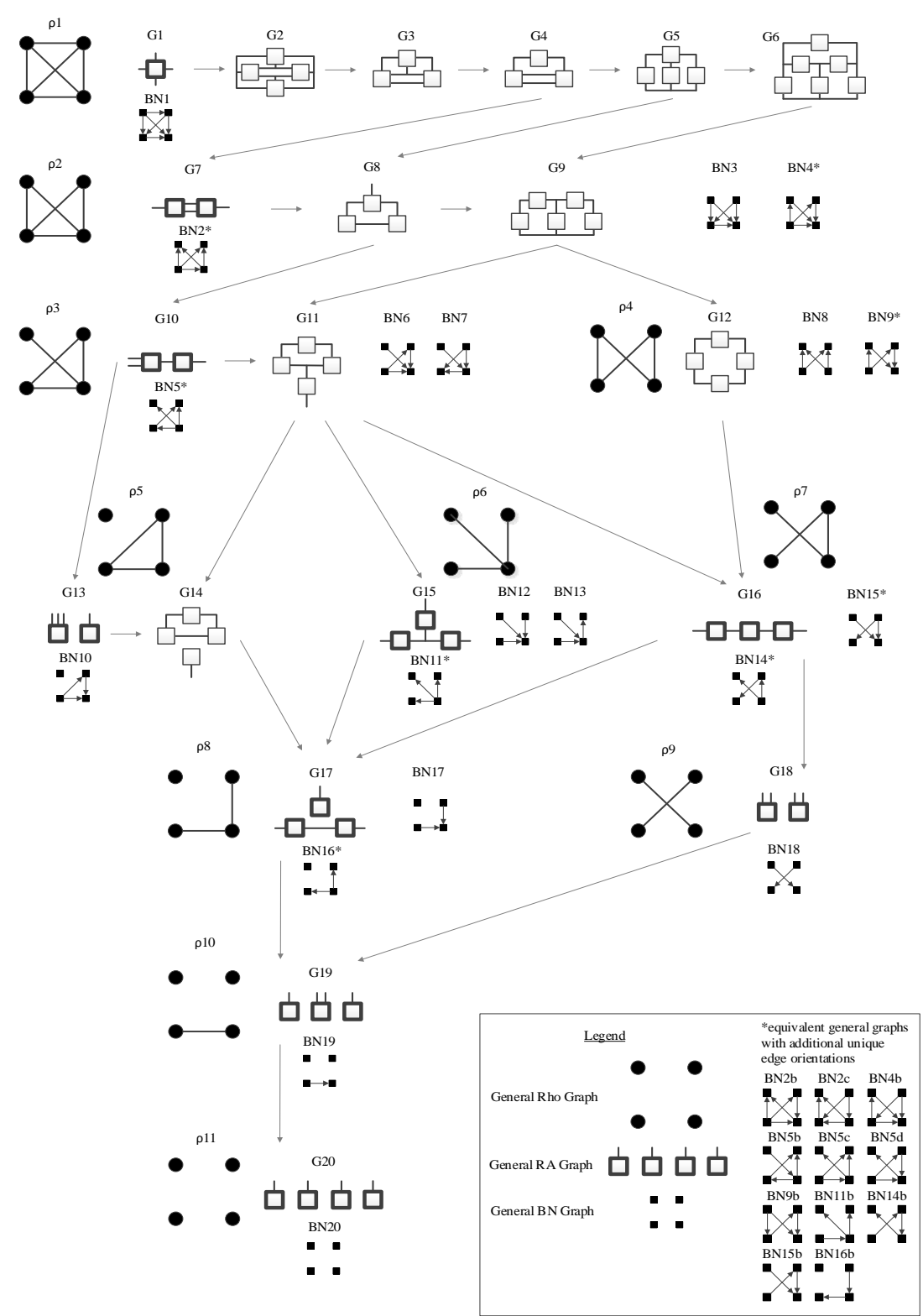

Fig. 5. Joint Lattice of RA and BN General Graphs

References(n.d.). 
Andersson, S. M. (1997). A Characterization of Markov Equivalence Classes For Acyclic Graphs. The annals of statistics.

Ashby, W. R. (1964). Constraint Analysis of Many-Dimensional Relations. General Systems Yearbook, 9, 99-105.

Bouckaert, R. (1994). Properties of Bayesian Belief Network Learning Algorithms. Uncertainty Proceedings, (pp. 102-109).

Broekstra, G. (1979). Nonprobabilistic constraint analysis and a two stage approximation method of structure identification. Proceedings of the 23rd Annual SGSR Meeting.

Buntine, W. (1991). Classifiers: A theoretical and empirical study. In Proceedings IJCAI (pp. 638-644). Sydney: Morgan Kaufmann.

Buntine, W. (1991). Theory refinement on Bayesian networks. Proceedings of the Seventh Conference on Uncertainty in Artificial Intelligence, (pp. 52-60). Los Angeles, CA.

Cavallo, R. (1979). The role of system science methodology in social science research. Martinus Nijhoff Publishing.

Chen, Y. (2016). Structure Discovery in Bayesian Networks: Algorithms and Applications. lowa State University graduate thesis.

Chickering, D. (1995). Transformational Characterization of Equivalent Bayesian Network Structures. Proceedings of the Eleventh Conference on Uncertainty in Artificial Intelligence, (pp. 87-98).

Chickering, D. (2002). Learning Equivalence Classes of Bayesian-Network Structures. Journal of Machine Learning Research.

Chickering, D. G. (1995). Learning Bayesian networks: Search methods and experimental results. In Proceedings of the Fifth Conference on Artificial Intelligence and Statistics (pp. 112-128). Ft. Lauderdale, FL: Society for Artificial Intelligence and Statistics. 
Chickering, D. H. (1997). A Bayesian approach to learning Bayesian networks with local structure. In D. a. Geiger (Ed.), Proceedings of the Thirteenth Conference on Uncertainty in Artificial Intelligence (pp. 8090). Providence, RI: Morgan Kaufmann.

Chickering, D. H. (2002). Large-Sample Learning of Bayesian Networks is NPHard. Journal of Machine Learning Research, 1287-1330.

Conant, R. (1981). Mechanisms of intelligence: Ashby's writings on cybernetics. Intersystems publications.

Conant, R. (1988). Extended dependency analysis of large systems. International journal of general systems.

Cooper, D. a. (1992). A Bayesian method for the induction of probabilistic networks from data. Machine Learning, 9:309-347.

Friedman, N. G. (1996). Building classifiers using Bayesian Networks. AAAl-96 Proceedings.

Friedman, N. K. (2003). Being Bayesian about network structure: A Bayesian approach to structure discovery in Bayesian networks. Machine Learning, 50(1-2):95-125.

Gillispie, S. a. (2001). Enumerating Markov Equivalence Classes of Acyclic Digraph Models. Proceedings of the Seventeenth conference on Uncertainty in Artificial Intelligence, (pp. 171-177).

Heckerman, D. G. (1994). Learning Bayesian networks: The combination of knowledge and statistical data. In proceedings of Tenth Conference on Uncertainty in Artificial Intelligence (pp. 293-301). Seattle, WA: Morgan Kaufmann.

Klir, G. (1976). Identification of generative structures in empirical data. International Journal of General Systems, 89-104.

Klir, G. (1985). The architecture of systems problem solving. New York: Plenum Press. 
Klir, G. (1986). Reconstructability analysis: An offspring of Ashby's constraint theory. Systems Research, 267-271.

Koivisto, M. a. (2004). Exact Bayesian structure discovery in Bayesian networks. Journal of Machine Learning Research, 549-573.

Krippendorff, K. (1981). An algorithm for identifying structural models of multivariate data. International Journal of General Systems, 63-79.

Krippendorff, K. (1986). Information Theory: Structural Models for Qualitative Data. Quantitative Applications in the Social Sciences \#62. Beverly Hills: Sage.

Larranaga, P. K. (1996). Learning Bayesian network structures by searching for the best ordering with genetic algorithms. IEEE Transactions on Systems, Man and Cybernetics, 26(4):487-493.

Lauritzen, S. (1996). Graphical models. Oxford Statistical Science Series. New York: Oxford University Press.

Malone, B. Y. (2011). Memory-Efficient Dynamic Programming for Learning Optimal Bayesian Networks. Proceedings of the Twenty-Fifth AAAI Conference on Artificial Intelligence, (pp. 1057-1062).

MIT. (2015). D-Separation. Retrieved from http://web.mit.edu/jmn/www/6.034/d-separation.pdf

Murphy, K. (1998). A Brief Introduction to Graphical Models and Bayesian Networks. Retrieved from https://www.cs.ubc.ca/ murphyk/Bayes/bayes_tutorial.pdf

Neapolitan, R. (1989). Probabilistic reasoning in expert systems: theory and algorithms. New York: Wiley.

Pearl, J. (1985). Bayesian Networks: A Model of Self-Activated Memory for Evidential Reasoning. Proceedings of the 7th Conference of the Cognitive Science Society, (pp. 329-334). Irvine, CA. 
Pearl, J. (1988). Probabilistic reasoning in intelligent systems: Networks of Plausible Inference. San Francisco: Morgan Kaufmann Publishers, Inc.

Pearl, J. (2000). Causality: Models, Reasoning, and Inference. Cambridge University Press.

Pearl, J. a. (1987). The logic of representing dependencies by directed graphs. Proceedings of the 6th National Conference on Artificial Intelligence. Seattle, WA.

Studený, M. V. (2010). Geometric view on learning Bayesian Network Structures. International Journal of Approximate Reasoning.

Tang, Y. S. (2012). Efficient and Accurate Learning of Bayesian Networks using Chi-Squared. Proceedings of the 21st International Conference on Pattern Recognition. Tsukuba, Japan.

Tian, J. H. (2010). Bayesian model averaging using the k-best Bayesian network structures. Proceedings of the Twenty-Sixth Conference on Uncertainty in Artificial Intelligence.

Verma, T. P. (1990). Equivalence and synthesis of causal models. Proceedings of the Sixth Annual Conference on Uncertainty in Artificial Intelligence, (pp. 255-270).

Wright, S. (1921). Correlation and causation. Journal of Agricultural Research. Journal of Agricultural Research, 557-585.

Wright, S. (1934). The method of path coefficients. The Annals of Mathematical Statistics, Vol. 5, No. 3, 161-215.

Zhang, H. (2004). The Optimality of Naive Bayes. . FLAIRS conference.

Zwick. (2010). Reconstructability Analysis of Epistasis. Annals of human genetics, 157-171. doi:10.1111/j.1469-1809.2010.00628.x

Zwick, M. (2001). Wholes and parts in general systems methodology. In: The character concept in evolutionary biology. (G. Wagner, Ed.) New 
York: Academic Press. Retrieved from

http://www.sysc.pdx.edu/download/papers/wholesg.pdf

Zwick, M. (2004). An overview of reconstructability analysis. Kybernetes, 33, 887-905. Retrieved from

http://www.sysc.pdx.edu/download/papers/ldlpitf.pdf

Zwick, M. C. (2018). Exploratory Reconstructability Analysis of Accident TBI Data. International Journal of General Systems, 47 (2): 174-191.

doi:10.1080/03081079.2017.1412435 